\title{
Transverse Instability Digital Damper for the Recycler
}

\author{
V.Balbekov \\ Fermi National Accelerator Laboratory \\ P.O. Box 500, Batavia, Illinois 60510
}

March 1, 2006

\begin{abstract}
Transverse beam instability of a coasting beam with a digital damper is examined. Threshold of instability is calculated in specific cases with Landau damping taken into account. The results are applied to the Fermilab Recycler Ring. Some improvement of existing $\mathrm{RR}$ damper is proposed.
\end{abstract}

\section{Introduction}

Transverse instability damper of the Fermilab Recycler Ring includes analogdigital and digital-analog converters with digital delay lines between [1]. Because the converters have own (sampling) frequency $52.83 \mathrm{MHz}$ (588 times the beam revolution frequency) they create a coupling of the beam eigenfrequencies. As a result, eigenmodes of the system can contain several frequencies even in the beam rest frame. This phenomenon not included in standard theory can effect on the beam instability changing performances of the damper. The problem was investigated first in Ref. [2]. Somewhat different methodics is used in this paper to investigate stability of a coasting beam giving special attention to Landau damping. 


\section{Damper transfer function and impedance}

Transformation of a signal by a pattern: Analog-Digital Converter - Delay Line - Digital-Analog Converter is schematically shown in Fig.1. Input signal $V^{(i n)}$ (black line) is measured with interval $T^{(c)} / M$ where $M$ is integer ( $M=4$ for the RR) and converted in digital form by ADC (blue points). Four consecutive numbers are averaged (green points), transferred with delay $T^{(d)}$ (red points), and converted by DAC to a stepped signal $V^{(o u t)}$ (red line). Thus

$$
V^{(\text {out })}(t)=\frac{1}{M} \sum_{m=0}^{M-1} V^{(i n)}\left(T^{(c)}[n-m / M]-T^{(d)}\right) \quad \text { at } \quad(n-1) T_{c}<t-T^{(d)}<n T^{(c)}
$$

Corresponding relation of Laplace images is:

$$
\begin{gathered}
\hat{V}^{(\text {out })}(\omega)=\int_{0}^{\infty} V^{(\text {out })}(t) \exp (i \omega t) d t= \\
\frac{\exp \left(i \omega T^{(c)}\right)-1}{2 \pi i \omega M} \int_{-\infty}^{\infty} \frac{\left[1-\exp \left(i \omega^{\prime} T^{(c)}\right] \exp \left(i \omega^{\prime} T^{(d)}\right) \hat{V}^{(i n)}\left(\omega^{\prime}\right) d \omega^{\prime}\right.}{\left[1-\exp \left(i \omega^{\prime} T^{(c)} / M\right)\right]\left[1-\exp \left(i\left[\omega-\omega^{\prime}\right] T^{(c)}\right)\right]}
\end{gathered}
$$

The under-integral function has poles at $\omega^{\prime}=\omega+n \omega^{(c)}$ which should be bypassed below resulting:

$$
\begin{gathered}
\hat{V}^{(\text {out })}(\omega)=\frac{2 \exp \left(i \omega T^{(c)}\right) \sin ^{2}\left(\omega T^{(c)} / 2\right)}{\omega M T^{(c)}} \times \\
\sum_{n} \frac{\exp \left(i\left[\omega+n \omega^{(c)}\right]\left[T^{(d)}-T^{(c)} / 2 M\right]\right)}{\sin \left(\left[\omega+n \omega^{(c)}\right] T^{(c)} / 2 M\right)} \hat{V}^{(i n)}\left(\omega+n \omega^{(c)}\right)
\end{gathered}
$$

where $\omega^{(c)}=2 \pi / T^{(c)}$. Eqiuvalent formula at $T^{(d)}=0$ was obtained in Ref. [2] where delay line was not included between AD and DA converters.

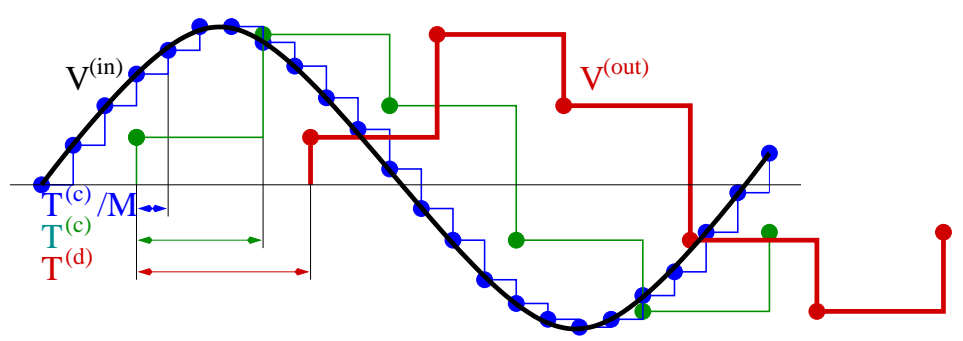

Figure 1: Schematic of AD-DA Conversion with delay line. 
The input signal is proportional to beam deviation on the pickup azimuthal position $\theta^{(p)}$ :

$$
\hat{V}^{(i n)}(\omega) \propto \hat{X}\left(\omega, \theta^{(p)}\right)=\sum_{k} \hat{X}_{k}(\omega) \exp \left(i k \theta^{(p)}\right)
$$

The output voltage is applied to a kicker located on azimuth $\theta^{(k)}$ and creates a $\delta$-shape force, i.e. it has a uniform Fourier spectrum:

$$
\hat{E}_{k}^{(d)}(\omega) \propto \hat{V}^{(\text {out })}(\omega) \exp \left(-i k \theta^{(k)}\right)
$$

Space between the kicker and pickup is

$$
\theta^{(p k)}=\theta^{(k)}-\theta^{(p)}=\frac{\pi[l+1 / 2]}{Q_{0}},
$$

where $Q_{0}$ is central betatron frequency and $l$ is integer. Two delay lines should be used between the pickup and the kicker:

$$
T^{(d)}=\frac{\theta^{(p k)}}{\Omega_{0}}-\frac{2 M-1}{2 M} T^{(c)} \quad \text { and } \quad T^{(d *)}=T^{(d)}+\frac{2 \pi}{\Omega_{0}}
$$

where $\Omega_{0}$ is the beam angular velocity. The signals are subtracted resulting the kicker field which, with Eq. (9) taken into account, can be represented in Fourier-Laplace domain as:

$$
\begin{aligned}
& \hat{E}_{k}^{(d)}(\omega)=i(-1)^{l} \frac{2 m \gamma \Omega_{0} Q_{0}}{e} \exp \left(i \theta^{(p k)}\left[\frac{\omega}{\Omega_{0}}-k\right]\right) \sum_{n} W_{n}^{(d)}(\omega) \sum_{k^{\prime}} \hat{X}_{k^{\prime}}\left(\omega+n \omega^{(c)}\right) \\
& W_{n}^{(d)}(\omega)=-i \mathcal{F}(\omega) \frac{2 \sin ^{2}\left(\omega T^{(c)} / 2\right) \exp \left(i n \theta^{(p k)} \omega^{(c)} / \Omega_{0}\right)}{\omega M T^{(c)} \sin \left(\left[\omega+n \omega^{(c)}\right] T^{(c)} / 2 M\right)}\left[1-\exp \left(2 \pi i \frac{\omega+n \omega^{(c)}}{\Omega_{0}}\right)\right]
\end{aligned}
$$

where $e$ and $m$ are the particle charge and mass, $\gamma$ is its normalized energy, and $\mathcal{F}(\omega)$ is gain produced by analog part of the damper (low-pass filter). We will see later that $W^{(d)}$ is decrement created by the damper.

Also we need to include field $E^{(z)}$ due to space charge, resistive wall, etc., symbolizing it as

$$
\hat{E}_{k}^{(z)}(\omega)=\frac{2 m \gamma \Omega_{0} Q_{0}}{e} W^{(z)}(\omega) \hat{X}_{k}(\omega)
$$

where the factor $W^{(z)}$ is proportional to the usual beam coupling impedance and numerically coincides with the impedance produced frequency shift. 


\section{Dispersion equation w/o Landau damping}

A spread of angular velocity and betatron tune of particles is neglected in this section. Then betatron oscillations of the beam center can be described by the equation:

$$
\left[\left[\frac{\partial}{\partial t}+\Omega_{0} \frac{\partial}{\partial \theta}\right]^{2}+\Omega_{0}^{2} Q_{0}^{2}\right] X(t, \theta)=\frac{e}{m \gamma}\left[E^{(z)}(t, \theta)+E^{(d)}(t, \theta)\right]
$$

It has a solution $\quad \hat{X}_{k}(\omega) \exp (i k \theta-i \omega t) \quad$ where:

$$
\hat{X}_{k}(\omega)=-\frac{e}{m \gamma} \frac{\hat{E}_{k}^{(z)}(\omega)+\hat{E}_{k}^{(d)}(\omega)}{\left[\omega-k \Omega_{0}\right]^{2}-\Omega_{0}^{2} Q_{0}^{2}}
$$

Using Eq. (8) and (10) one can represent it in the form:

$$
\hat{X}_{k}(\omega)=i(-1)^{l+1} \exp \left(i \theta^{(p k)}\left[\frac{\omega}{\Omega_{0}}-k\right]\right) \frac{2 \Omega_{0} Q_{0} \sum_{n} W_{n}^{(d)}(\omega) \sum_{k^{\prime}} \hat{X}_{k^{\prime}}\left(\omega+n \omega^{(c)}\right)}{\left[\omega-k \Omega_{0}\right]^{2}-\Omega_{0}^{2} Q_{0}^{2}+2 \Omega_{0} Q_{0} W^{(z)}(\omega)}
$$

Summarizing over $k$ results:

$$
\hat{X}(\omega)=S(\omega) \sum_{n} W_{n}^{(d)}(\omega) \hat{X}\left(\omega+n \omega^{(c)}\right)
$$

where $\hat{X}(\omega)=\sum_{k} \hat{X}_{k}(\omega)$ and

$$
S(\omega)=\sum_{k} \frac{2 i(-1)^{l+1} \Omega_{0} Q_{0} \exp \left(i \theta^{(p k)}\left[\omega / \Omega_{0}-k\right]\right)}{\left[\omega-k \Omega_{0}\right]^{2}-\Omega_{0}^{2} Q_{0}^{2}+2 \Omega_{0} Q_{0} W^{(z)}(\omega)}
$$

As usually, unperturbated spectrum of the beam includes frequencies:

$$
\omega_{k}^{(\mp)}=\Omega_{0}\left(k \mp Q_{0}\right)
$$

among which there are not coinciding because $Q_{0}$ can't be integer or halfinteger. However, the digital damper produces a coupling of the harmonics which depends on the parameter $\left(\omega^{(c)} / \Omega_{0}\right.$ - nearest integer $)$. We consider the ultimate cases separately. 


\section{$3.1 \quad$ Non-integer $\omega_{c} / \Omega$ (offset damper)}

If a deviation of $\omega_{c} / \Omega$ from integer is rather large, only term $n=0$ gives a contribution is series (14) providing dispersion equation $S(\omega) W_{0}^{(d)}(\omega)=1$. Expression (15) can be simplified also:

$$
S(\omega) \simeq \frac{1}{\omega-\omega_{k}^{(\mp)} \mp W^{(z)}\left(\omega_{k}^{(\mp)}\right)}
$$

As a result, solution of the dispersion equation is:

$$
\omega \simeq \omega_{k}^{(\mp)}+W^{(t)}\left(\omega_{k}^{(\mp)}\right)
$$

where

$$
W^{(t)}\left(\omega_{k}^{(\mp)}\right)= \pm W^{(z)}\left(\omega_{k}^{(\mp)}\right)+W_{0}^{(d)}\left(\omega_{k}^{(\mp)}\right)
$$

It means that the beam coupling impedance and the damper contribution are additive in this case. Somewhat simplifying Eq. (9), one can represent the damper contribution in the form:

$$
W_{0}^{(d)}(\omega) \simeq-i \Delta \omega^{(d)}\left[\frac{\sin \left(\pi \omega / \omega^{(c)}\right)}{\pi \omega / \omega^{(c)}}\right]^{2} \mathcal{F}(\omega)
$$

where the constant $\Delta \omega^{(d)}$ is the damper decrement at low frequency. Sometimes we will use a simplest model of the low-pass filter:

$$
\mathcal{F}(\omega)=\left\{\begin{array}{lll}
1 & \text { at } & |\omega|<\omega^{(f)} \\
0 & \text { at } & |\omega|>\omega^{(f)}
\end{array}\right\}
$$

Then one can assert that the offset damper is capable to provide the beam stability overall in the band $|\omega|<\min \left\{\omega^{(c)}, \omega^{(f)}\right\}$, i.e. $|\omega|<\omega^{(c)}$, at reasonably wide-band filter.

The damper offset should be more of the beam frequency spread to avoid an overlapping of Schottky bands. An optimal offset is obtained if space between $\omega^{(c)} / \Omega_{0}$ and nearest integer number coincides with space between $Q_{0}$ and nearest half-integer.

As for threshold of instability, frequency spread and Landau damping should be taken into account to determine it, which will be done in next section. 


\subsection{Integer $\omega^{(c)} / \Omega_{0}$, narrow-band filter}

The ratio $\omega^{(c)} / \Omega_{0}$ is integer and bandwidth of the filter satisfies the condition $\omega^{(c)} / 2<\omega^{(f)}<\omega^{(c)}$ at the existing RR damper [1]. This case is considered here still without Landau damping. Then series (14) is reduced to 2 equations:

$$
\begin{aligned}
& \hat{X}(\omega)=S(\omega)\left[W_{0}^{(d)}(\omega) \hat{X}(\omega)+W_{s}^{(d)}(\omega) \hat{X}\left(\omega+s \omega^{(c)}\right)\right] \\
& \hat{X}\left(\omega+s \omega^{(c)}\right)=S\left(\omega+s \omega^{(c)}\right)\left[W_{0}^{(d)}\left(\omega+s \omega^{(c)}\right) \hat{X}\left(\omega+s \omega^{(c)}\right)+W_{-s}^{(d)}\left(\omega+s \omega_{c}\right) \hat{X}(\omega)\right]
\end{aligned}
$$

where $s=-\operatorname{sgn}(\omega)$. Condition of its solveability (dispersion equation) is:

$$
\begin{gathered}
{\left[1-S(\omega) W_{0}^{(d)}(\omega)\right]\left[1-S\left(\omega+s \omega^{(c)}\right) W_{0}^{(d)}\left(\omega+s \omega^{(c)}\right)\right]} \\
=S(\omega) S\left(\omega+s \omega^{(c)}\right) W_{s}^{(d)}(\omega) W_{-s}^{(d)}\left(\omega+s \omega^{(c)}\right)
\end{gathered}
$$

Using Eq. (9) and a simplifying $S(\omega)$ like (17), one can represent it in the form:

$$
\left[W-W^{(t)}\left(\omega_{k}^{(\mp)}\right)\right]\left[W-W^{(t)}\left(\omega_{k}^{(\mp)}+s \omega^{(c)}\right)\right]=W^{(d)}\left(\omega_{k}^{(\mp)}\right) W^{(d)}\left(\omega_{k}^{(\mp)}+s \omega^{(c)}\right)
$$

where $W=\omega-\omega_{k}^{(\mp)}$, and $W^{(t)}(\omega)$ is given by Eq. (19). It is seen that the coupling of harmonics really exists at $\omega^{(c)}-\omega^{(f)}<|\omega|<\omega^{(f)}$ only; otherwise the right-hand part of Eq. (24) is zero, and we turn back to solutions (18).

We consider the beam behavior in the coupling region in detail, describing the damper by simplified formulae (20)-(21) and assuming that resistive wall impedance is a source of instability:

$$
W^{(z)}(\omega)=\Delta \omega^{(r w)}[1+i \operatorname{sgn}(\omega)] \sqrt{\frac{\Omega_{0}}{|\omega|}}
$$

Solution is displayed in left Fig. 1 at $\Delta \omega^{(r w)}=0.0003 \Omega_{0}, \Delta \omega^{(d)}=$ $0.0005 \Omega_{0}$, and $\omega^{(c)}=588 \Omega_{0}, \omega^{(f)}=400 \Omega_{0} \quad(52.8 \mathrm{MHz}$ and $35.9 \mathrm{MHz}$ for the RR). Upper sign in the combination $(\mp)$ taken for the definiteness, because opposite choice means simply a mirror reflection of all traces without additional physical content. Red and blue traces present imaginary part of 2 eigenvalues against the basic frequency $\omega_{k}$. Because the satellite frequency

$\omega_{k}+s \omega^{(c)}$ assists at the spectrum also, the green trace shows ration of the 

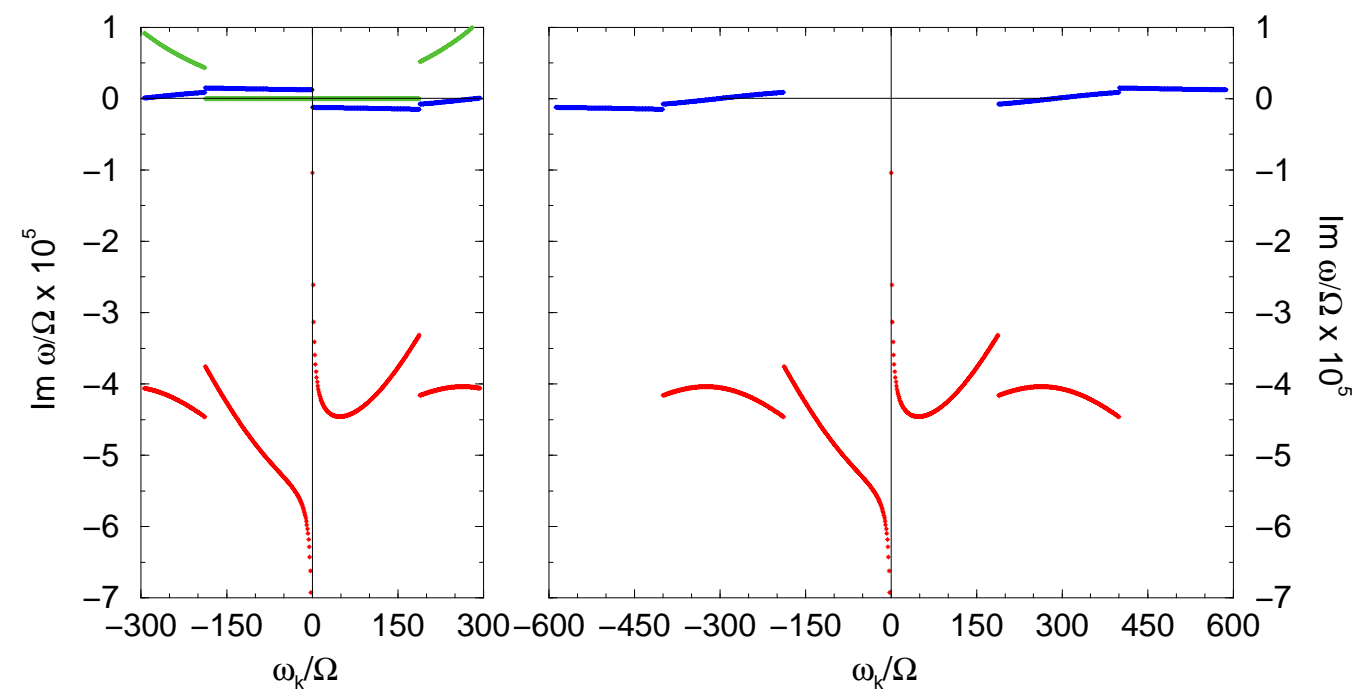

Figure 2: Imaginary part of eigenfrequencies at $\Delta \omega^{(r w)} / \Omega_{0}=$ $0.0003, \Delta \omega^{(d)} / \Omega_{0}=0.0005$. Only lowest frequency is used in the left graph, and both physical frequencies in the right one.

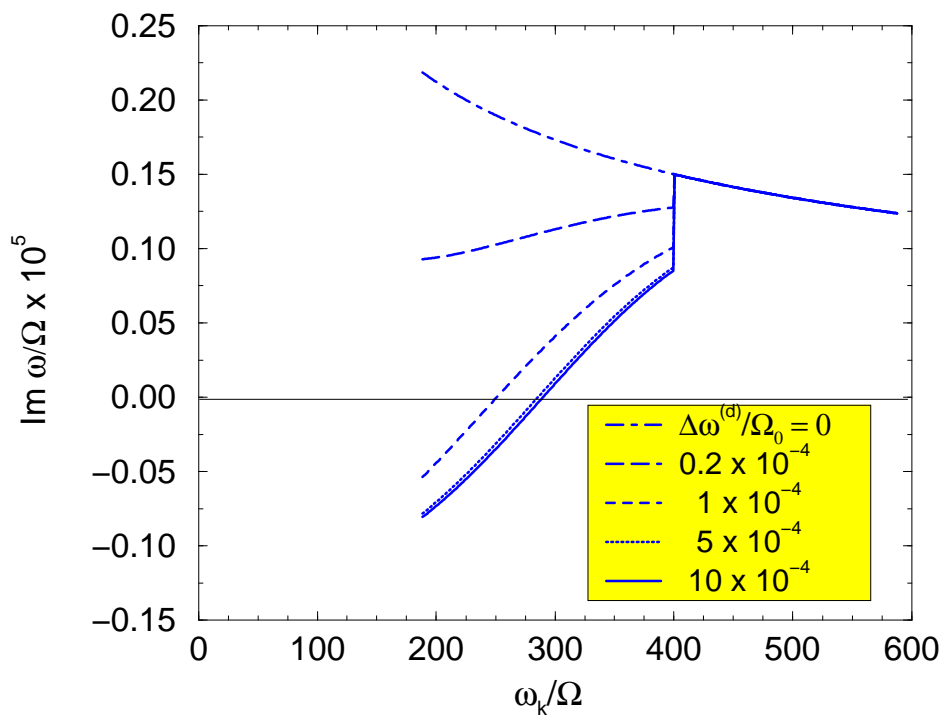

Figure 3: Imaginary part of eigenfrequencies at $\Delta \omega^{(r w)} / \Omega_{0}=0.0003$ and different $\Delta \omega^{(d)} / \Omega_{0}=0.0005$. Only potentially unstable solution is shown. 
amplitudes: satellite/basic for the 'red' solution and basic/satellite for 'blue' one. As expected, there is sole frequency (18) at $\left|\omega_{k}\right|<\omega^{(c)}-\omega^{(f)}$ : basic (lowest) for the 'red' case and satellite for the 'blue' one. It is more apparent by right Fig. 2 where the traces are partly shifted on 588 points to make visible real frequencies as possible ('long scale').

Note that the 'red' solution strongly depends on the damper gain, moving proportionally up or down. 'Blue' one changes rather weakly as it is shown in Fig. 3 where corresponding imaginary part is represented in 'long' scale at $\Delta \omega^{(r w)}=0.0003 \Omega_{0}$ and different $\Delta \omega^{(d)}$.

\section{Landau damping and threshold of instability}

Positive imaginary part of frequency obtained in previous section means only a possibility of instability, which can be suppressed by Landau damping in reality. Spreads of revolution frequency and betatron tune should be added to Eq. (11) to include this effect. A formal replacement in Eq. (12) and later follows by this:

$$
\frac{1}{\left[\omega-k \Omega_{0}\right]^{2}-\Omega_{0}^{2} Q_{0}^{2}} \Rightarrow \int_{-\infty}^{\infty} \frac{F(p) d p}{[\omega-k \Omega(p)]^{2}-\Omega^{2}(p) Q^{2}(p)}
$$

where $F(p)$ is normalized distribution function on momentum. Then the basic series of equations (14) holds its form with correspondingly modified function $S(\omega)$. Besides of this, we need to modify definition (24) to include space charge impedance:

$$
W^{(z)}(\omega)=\Delta \omega^{(s c)}+\Delta \omega^{(r w)}[1+i \operatorname{sgn}(\omega)] \sqrt{\frac{\Omega_{0}}{|\omega|}}
$$

Real value $\Delta \omega^{(s c)}$ depends on the beam density, and for Gaussian beam it is [3]

$$
\Delta \omega^{(s c)}=\frac{r_{0} \Omega_{0} N}{8 \pi \beta \gamma^{2} \epsilon_{\perp}}
$$

where $r_{0}=e^{2} / m c^{2}\left(1.535 \times 10^{-16} \mathrm{~cm}\right.$ for protons $), N$ is number of particles, and $\epsilon_{\perp}$ is transverse r.m.s. emittance. This addition would cause only a general real shift of all frequencies in previous section. However, with Landau damping it is the main factor determining threshold of instability. 


\subsection{Non-integer $\omega_{c} / \Omega$}

In this case there is no coupling of the beam eigenmodes, and dispersion equation is reduced to a standard form:

$$
1=W^{(t)}\left(\omega_{k}^{(\mp)}\right) \int_{-\infty}^{\infty} \frac{F(p) d p}{W-\delta \omega_{k}^{(\mp)}\left[p-p_{0}\right] / \sigma_{p}}
$$

where

$$
\delta \omega_{k}^{(\mp)}=\frac{d}{d p}[\Omega(p)[k \mp Q(p)]] \sigma_{p}
$$

is r.m.s. frequency spread, and definition (19) is used. For Gaussian beam in

practical case $\operatorname{Re} W^{(t)}>\operatorname{Im} W^{(t)}$, approximate (not worse of $20 \%$ ) condition of stability is:

$$
\left|\frac{\operatorname{Re} W^{(t)}\left(\omega_{k}^{(\mp)}\right)}{\delta \omega_{k}^{(\mp)}}\right|<\sqrt{\ln \left(\frac{4}{3}\left|\frac{\operatorname{Re} W^{(t)}\left(\omega_{k}^{(\mp)}\right)}{\operatorname{Im} W^{(t)}\left(\omega_{k}^{(\mp)}\right)}\right|^{2}\right)}
$$

We will apply it to the RR taking upper sign in the combination $(\mp)$ and using numerical values:

- Resistive wall tune shift:

$$
\frac{\Delta \omega^{(r w)}}{\Omega_{0}}=0.5 \times 10^{-6}\left(\mathrm{~N} / 10^{10}\right)
$$

- Space charge tune shift:

$$
\frac{\Delta \omega^{(s c)}}{\Omega_{0}}=6.8 \times 10^{-6} \frac{N / 10^{10}}{\epsilon_{\perp}(\mathrm{mm}-\mathrm{mrad})}
$$

- Tune spread:

$$
\frac{\delta \omega_{k}}{\Omega_{0}}=10 \times 10^{-6}\left|0.0085\left(k-Q_{0}\right)-\xi\right| \epsilon_{\|}(\mathrm{eV}-\mathrm{s})
$$

where $\xi$ is chromaticity and $\epsilon_{\|}$is longitudinal r.m.s. emittance. Because transverse r.m.s. emittance in the RR certainly less of $10 \mathrm{~mm}-\mathrm{mrad}$, space charge impedance dominates, what allows to represent stability condition (31) in the form:

$$
D<0.061\left|0.0085\left[k-Q_{0}\right]-\xi\right| \sqrt{\ln \left(\frac{250\left[k-Q_{0}\right]}{\epsilon_{\perp}^{2}(\mathrm{~mm}-\mathrm{mrad})}\right)}
$$


where $D$ is the beam 'phase density' in terms of $95 \%$ emittances:

$$
D=\frac{N / 10^{10}}{\left[4 \epsilon_{\|}(\mathrm{eV}-\mathrm{s})\right]\left[6 \epsilon_{\perp}(\mathrm{mm}-\mathrm{mrad})\right]}
$$

A rough estimation of the threshold density can be obtained using a typical emittance $\epsilon_{\perp} \sim 1 \mathrm{~mm}$-mrad in the right-hand part of Eq. (35). More exact results are plotted in Fig. 4 at nominal chromaticity $\xi=-2$ and at $\xi=-6$. Some numerical solutions of Eq. (29) without approximation (31) are shown also demonstrating rather good agreement. The lowest harmonic $k-Q_{0}=0.575$ ( $k=1$ in the picture) is most unstable if the damper is off. If the damper is on and its gain is large enough, threshold of instability can be obtained also by Eq. (35) at $k-Q \simeq 588$ (it is presumed that min $\left.\left\{\omega^{(f)}, \omega^{(c)}\right\}=588 \Omega_{0}\right)$. It is seen from the figure that the offset damper can provide merit factor $5-9$ at nominal chromaticity $\xi=-2$ and $3-5$ at $\xi=-6$.

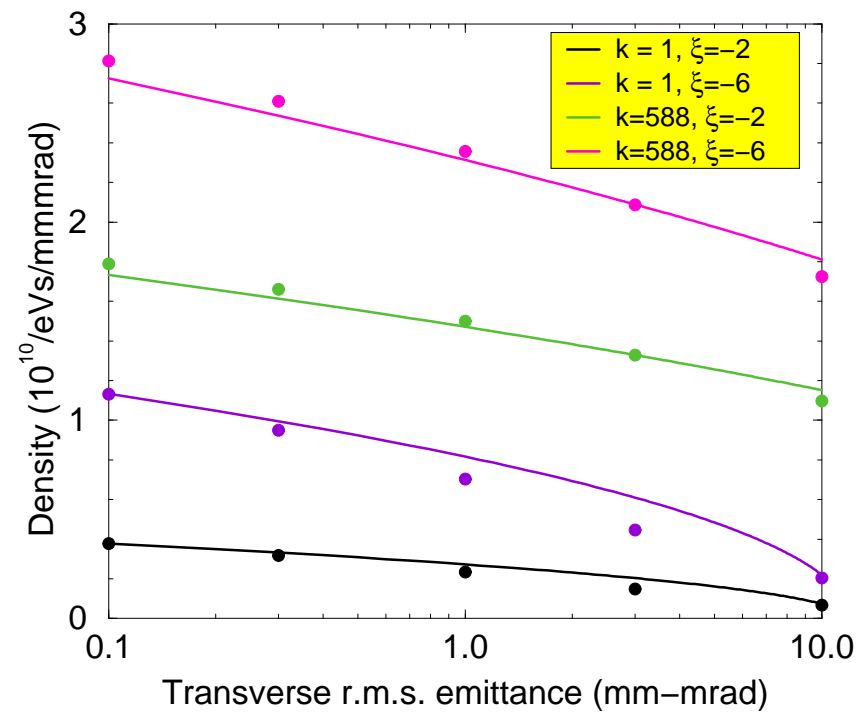

Figure 4: Threshold beam density vs transverse emittance. Solid lines Eq. (35), circles - numerical solution of Eq. (29) 


\subsection{Integer $\omega^{(c)} / \Omega_{0}$, narrow-band filter}

In this part, we are assuming again that the damper characteristic is given by Eq. (20) and $(21)$ at $\omega^{(c)} / 2<\omega^{(f)}<\omega^{(c)}$. With Landau damping taken into account, it gives the following dispersion equation instead of (23):

$\left[I_{k, 0}^{(\mp)}-W^{(t)}\left(\omega_{k}^{(\mp)}\right)\right]\left[I_{k, s}^{(\mp)}-W^{(t)}\left(\omega_{k}^{(\mp)}+s \omega^{(c)}\right)\right]=W^{(d)}\left(\omega_{k}^{(\mp)}\right) W^{(d)}\left(\omega_{k}^{(\mp)}+s \omega^{(c)}\right)$

where

$$
I_{k, n}^{(\mp)}(W)=\left[\int_{-\infty}^{\infty} \frac{F(p) d p}{W-\delta \omega_{k, n}^{(\mp)}\left[p-p_{0}\right] / \sigma_{p}}\right]^{-1}
$$

and

$$
\delta \omega_{k, n}^{(\mp)}=\frac{d}{d p}\left[\Omega(p)\left[k+n \omega^{(c)} / \Omega_{0} \mp Q(p)\right]\right] \sigma_{p}
$$

Numerical solution of this equation is performed at the same conditions as above (see subsection 3.2, and Eq. (27), (32)-(34)), Damper gain providing $\Delta \omega^{(d)}=5 / 3 \Delta \omega^{(r w)}$ is taken to ensure suppression of all low-frequency harmonics. Ultimate phase density (36) is plotted in Fig. 5 vs harmonic number

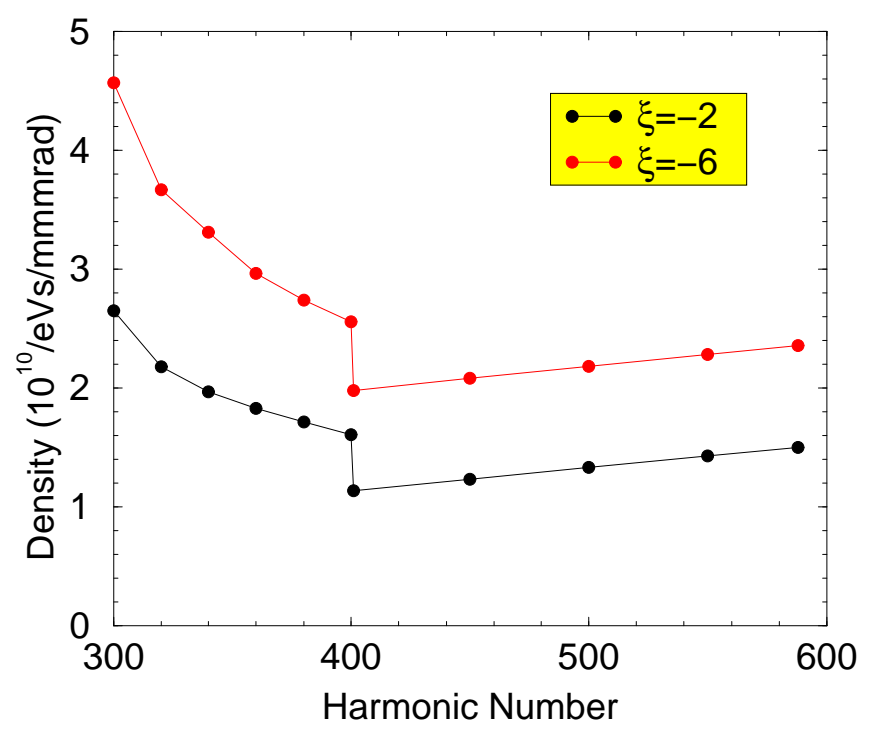

Figure 5: Threshold beam density vs transverse emittance. Solid lines Eq. (35), circles - numerical solution of Eq. (29) 
at $\epsilon_{\perp}=1 \mathrm{~mm}-\mathrm{mrad}$ and chromaticity -2 or -6 . The 'long' frequency scale is used in this case like Fig. 3.

For harmonics $k>400$, i.e. outside the damper band, the same result as above is obtained - see Eq. (35) and Fig. 4. An abruption at $k=400$ appears because of approximation (21) used, and would be smoothed out at more realistic filter characteristic. Then the density increases i.e.the beam turns more stable, reaching a total stabilization (formally infinite density) at $k<$ 286. There is a distinction from an offset damper in this point, because the last would assure a total stabilization even at $k<400$ at the same conditions (or $k<588$ with wider low-pass filter). Another distinction is that an additional gain of the onset damper effects on this (less stable) mode only slightly, as it is seen also from Fig. 4. Nevertheless, considered 'mediumband' onset damper is also capable to bring threshold of instability inside the band to a level exceeding the outside one.

\section{Conclusion}

It is shown that a digital damper, in principle, could provide a beam stability right up to sampling frequency, that is up to $52.8 \mathrm{MHz}$ in the Recycler. Of course, low-pass filter should be wide-band enough also. However, an excessive width can create problems at integer ratio of sampling/revolution frequencies, coupling more of 2 harmonics with unclear consequences. The damper offset cracks the problem bringing an optimal result if the filter band a bit more of the sampling frequency - about $60 \mathrm{MHz}$ for the Recycler. The offset providing maximal space between the Schottky bands is required in this case. It is about $7 \mathrm{kHz}$ (optimal) but not less of $1 \mathrm{kHz}$ for the RR.

Next table summarizes the RR digital damper efficiency at tune $0.425+$ integer and transverse r.m.s. emittance $1 \mathrm{~mm}$-mrad. Ultimate phase density of the beam determined by Eq. (36) as well as most unstable harmonic number are given for different cases.

\begin{tabular}{||c|c|c|c||}
\hline \hline Chromaticity & $\begin{array}{c}\text { Damper off } \\
\mathrm{k}=1\end{array}$ & $\begin{array}{c}\text { Damper on } \\
k \simeq 400\end{array}$ & $\begin{array}{c}\text { Upgraded damper } \\
k \simeq 588\end{array}$ \\
\hline \hline$\xi=-2$ & 0.23 & 1.1 & 1.5 \\
\hline$\xi=-6$ & 0.70 & 2.0 & 2.4 \\
\hline \hline
\end{tabular}




\section{References}

[1] J. Crisp, M. Hu, and V. Tupikov, FERMILAB-CONF-05-167-AD (May 2005).

[2] A. Burov, http://lss.fnal.gov/archive/test-tm/2000/fermilab-tm-2336ad.pdf

[3] V. Balbekov, FERMILAB-FN-0782-AD (January 2006). 